\title{
Effective Adaptive Radiotherapy for Locally Advanced Lung Cancer Treated with 3D Conformal Technique
}

\author{
Dina A. Salem ${ }^{1}$, Somaia M. El Sayed ${ }^{2}$, Amin E. A. Amin² \\ ${ }^{1}$ Clinical Oncology, Radiation Oncology and Nuclear Medicine Department, Faculty of Medicine, Ain \\ Shams University, Cairo, Egypt; ${ }^{2}$ Medical Physics, Clinical Oncology and Nuclear Medicine \\ Department, Faculty of Medicine, Ain Shams University, Cairo, Egypt
}

Background: The reduction in tumor volume during the treatment of locally advanced non-small cell lung cancer (NSCLC) with chemoradiotherapy allows the application of adaptive radiotherapy strategy. Adaptive planning has the potential for improving radiotherapy planning indices and dose escalation while respecting the dose to the organs at risk (OARs).

Aim: To evaluate the benefit of adaptive radiotherapy in patients with advanced NSCLC treated with conformal technique. Methods: In this dosimetric planning study, we included computerized tomography data-sets from 10 patients with locally advanced NSCLC who responded to the first 22 fractions (44 Gy) and achieved $>20 \%$ regression in gross tumor volume. We designed for each patient two plans. Plan 1 (conventional planning) in which the whole prescribed dose (64 Gy) was delivered to the planning target volume 1 (PTV1). Plan 2 (adaptive planning) was divided into plan 2A delivering 44 Gy to PTV1 and plan 2B delivering 20 Gy to PTV2. In plan 2B we re-delineated the PTV and all the target volumes on the repeated CT images to deliver the rest of the prescribed dose $(20 \mathrm{~Gy})$. Plan 2A and Plan $2 \mathrm{~B}$ were summed to measure the accumulated dose delivered to OARs.

Results: The mean PTV2 was significantly smaller than the mean PTV1 ( $p<0.001)$ with 53\% reduction. Adaptive planning resulted in $13.2 \%$ mean reduction in the maximum dose to the spinal cord $(p<0.001), 10.4 \%$ in esophagus $\mathrm{V} 35$ ( $<<0.001)$, $11.4 \%$ in esophagus V50 ( $<<0.001), 24.3 \%$ in mean lung dose $(\mathrm{p}=0.001)$ and $19.7 \%$ in lung V20 (p<0.001). The reduction in heart d-max and heart mean dose was not significant.

Conclusion: The adaptive radiotherapy technique used in our study can reduce the irradiated volume and minimize the dose delivered to OARs. This simple applicable technique can be easily implemented in limited resources centers.

Keywords: Adaptive radiotherapy, Non small cell lung cancer, Chemoradiotherapy.

Corresponding author: Dina Salem, MD, Clinical Oncology Department, Faculty of Medicine, Ain Shams University,

Cairo, Egypt, E-mail: dinasalem70@yahoo.com

Submitted: 11-September-2017; Revised: 23-October-2017; Accepted: 31-October-2017; Published online: 31-December-2017

\section{INTRODUCTION}

Lung cancer remains the most common cancer worldwide for several decades. The incidence of lung cancer is estimated to be $12.9 \%$ in $2012,58 \%$ of which occurred in developing countries 1. Concomitant chemoradiation remains the standard treatment of locally advanced lung cancer but it is associated with considerable toxicity ${ }^{2}$.

Reduction of toxicity of chemoradiation can be achieved by using smaller fields which is possible by approaches that eliminate elective mediastinal radiation and technologies such as four-dimensional computed tomography (CT) scans for planning and those using image guided delivery techniques ${ }^{3-5}$.

In developing countries, radiotherapy departments may not have the luxury of such advanced facilities. Can such centers adopt more simple strategies in radiotherapy planning to improve the conformity, allowing more reduction in doses to organs at risk (OARs) and allowing for dose escalation?

For early-stage NSCLC treated with hypofractionated stereotactic body radiotherapy,
Guckenberger et al observed a dose-response relationship for local control, cancer-specific survival, and overall survival. These data are the scientific basis for possibility of an escalation of the radiation dose using new technique such as adaptive radiotherapy ${ }^{6}$.

Adaptive radiotherapy is defined as adaptation of the treatment plan to changes of the tumor volume, shape and position based on three-dimensional (3D) imaging of lung tumor during the course of radiation to detect shrinkage of the gross tumor volume (GTV) and hence applying replanning strategy for effective tumor control with the possibility of dose escalation and decreasing dose to OARs. Optimization of chemoradiation with applicable simple strategies especially in the context of developing countries may help to overcome various challenges in the treatment of locally advanced NSCLC.

The concept of adaptive radiotherapy is based on the likely tumor regression of advanced NSCLC during the course of radiotherapy or chemoradiation 7,8 . This tumor regression makes approaches such as adaptive radiotherapy attractive. 
The aim of this study was to evaluate the potential benefit of adaptive radiotherapy treatment planning in reducing the irradiated tumor volume and in minimizing the radiation dose to OARs in a limited-resources setting.

\section{METHODS}

Ten patients with locally advanced NSCLC were included in this dosimetric planning study. The treatment plan for these patients was radical radiotherapy (64 Gy) with conventional fractionation ( 2 Gy per fraction) with weekly concomitant chemotherapy (paclitaxel $50 \mathrm{mg} / \mathrm{m}^{2}$ with carboplatin AUC2). For evaluation of the adaptive radiotherapy benefits, all patients were imaged on $\mathrm{CT}$ simulator (Clayton-Toshiba) as a pretreatment procedure for contouring of gross tumor volume (GTV), clinical target volume (CTV), planning target volume (PTV) and OARs (spinal cord, esophagus, heart and lung). At 44 Gy, CT scan was performed to identify patients with $>20 \%$ regression in GTV for inclusion in this study.

\section{Imaging}

All patients underwent CT scan simulation in the supine position on a CT scanner (Toshiba Clayton- wide bore). Slice thickness was $5 \mathrm{~mm}$ for all CT images. Diagnostic positron emission tomography (PET) scan was also performed and the images were fused with CT scans. All structures were delineated on the CT images. The GTV was delineated with guidance from PET-CT images (areas of high 18F-FDG uptake). Central necrosis within the tumor was not excluded from GTV. Lymph nodes were defined to be involved if they had high 18F-FDG uptake on PET images.

The CTV was generated by adding a 5-10 mm margin to the respective GTV in all dimensions. The PTV was generated with a $5 \mathrm{~mm}$ radial and $10 \mathrm{~mm}$ superior and inferior. CT scans were repeated after 44 Gy and similar steps were performed. Modification of the targets as well as normal tissue contours (ipsilateral lung) was implemented on the $2^{\text {nd }}$ scan to adapt to tumor volume change. For GTV, the lymph nodes considered significant on the baseline scan were identified and contoured irrespective of the size criteria.

\section{Planning}

All images were transferred to the TPS, Eclipse ${ }^{\mathrm{TM}} \mathrm{V}$. 13.5. (Varian Medical Systems, Palo Alto, CA) via Diacom network. The 3D conformal planning was performed for all patients and consisted of three to five fields with $6 \mathrm{MV}$ coplanar photon beams. Selection of beam angles depended on the position and the PTV in such that the beams would pass through a minimum amount of normal tissue before reaching the tumor and at the same time avoids collisions with the table and patient. Figure 1A shows the field arrangement of the applied 3D-conformal plan. The prescribed dose of 64 Gy had been delivered divided on 32 fractions in one phase. A dose distribution was considered acceptable for treatment if $100 \%$ of the PTV would get $95 \%$ of the prescribed dose. The dose to the spinal cord was limited to a maximum of $50 \mathrm{~Gy}$, the mean lung dose (excluding

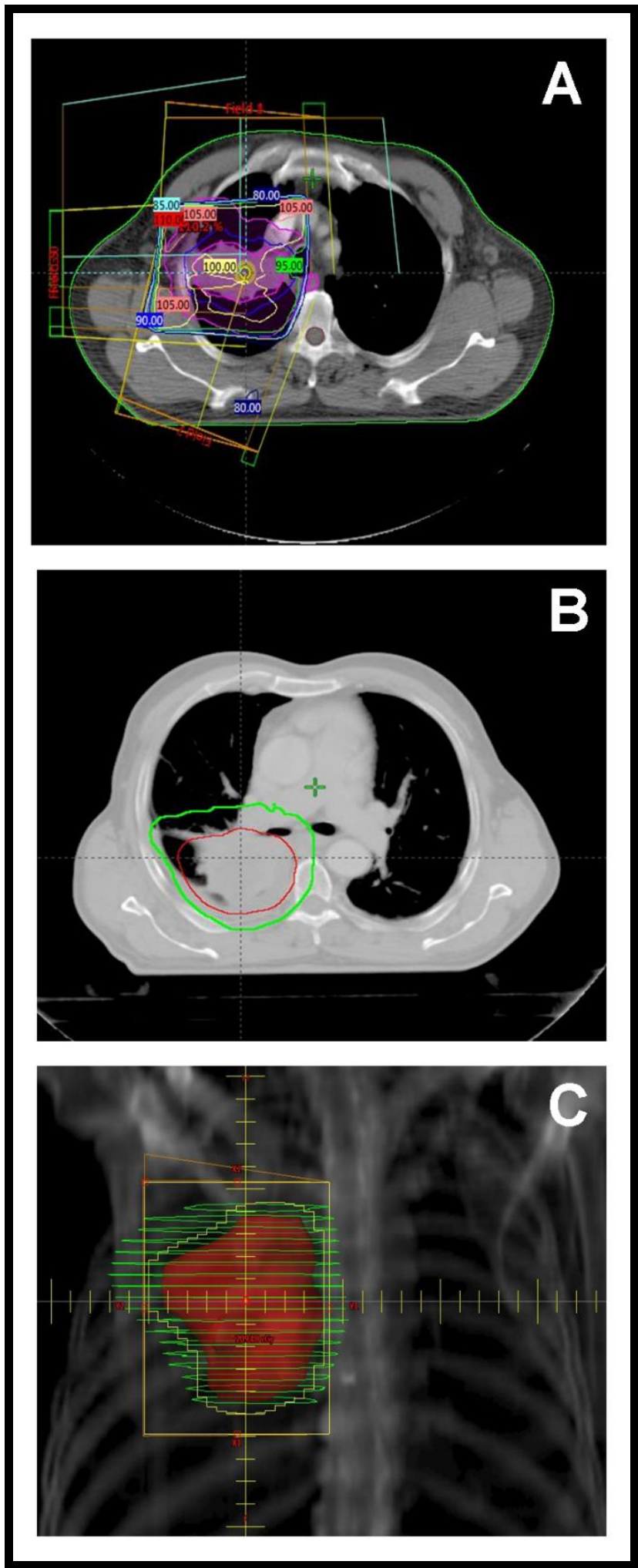

Figure 1. A: Fields arrangement applied for all plans (conventional and adaptive); B: PTV1 (green) and PTV2 (red); C: Volumetric reduction of the PTV.

GTV) to $<20$ Gy and lung V20 to $<35 \%$. Esophagus V35 dose was limited to $<65 \%$, esophagus V50 dose to $<40 \%$ and the mean heart dose to $<26 \mathrm{~Gy}$.

We designed for each patient two plans:

- Plan 1 (conventional planning): in which the prescribed dose had been delivered in one phase to each patient. 
- Plan 2 (adaptive planning) was divided into plan 2A delivering 44 Gy to PTV1, and plan 2B delivering $20 \mathrm{~Gy}$ to PTV2. In plan 2B similar steps were performed and the PTV1 was modified on the $2^{\text {nd }}$ scan to adapt to tumor volume change and to deliver the rest of the prescribed dose $20 \mathrm{~Gy}$. Figures $1 \mathrm{~B}$ and 1C show the PTV1 (green) which had been delineated on the first CT images and the PTV2 (red) which had been delineated on the $2^{\text {nd }} \mathrm{CT}$ images.

Differences in PTV, maximum dose of the spinal cord, V35 and V50 of the esophagus, maximum and mean doses of the heart, and V20 and mean dose of the lung in both planes (plane 1 and adaptive plan 2A and 2B) were analyzed and compared.

\section{Statistical Analysis}

Data were collected, revised, coded and entered to the Statistical Package for Social Science (IBM SPSS) version 23. Quantitative data were presented as mean \pm standard deviations and ranges when their distribution found parametric by Kolmogorov-Smirnov test of normality. The comparison between two paired groups with quantitative data and parametric distribution were done by using paired $t$-test. The confidence interval was set to $95 \%$ and the margin of error accepted was set to $5 \%$. So, the $p$ value was considered significant at $<0.05$.

\section{RESULTS}

The characteristics of the 10 patients included in this dosimetric study are summarized in table 1 .

Table 1. Patients' characteristics

\begin{tabular}{|c|c|}
\hline & $n(\%)$ \\
\hline \multicolumn{2}{|l|}{ Age } \\
\hline Median (range) & $57(50-67)$ \\
\hline \multicolumn{2}{|l|}{ Sex } \\
\hline Male & $8(80)$ \\
\hline Female & $2(20)$ \\
\hline \multicolumn{2}{|l|}{ T stage } \\
\hline $\mathrm{T} 2$ & $1(10)$ \\
\hline T3 & $6(60)$ \\
\hline $\mathrm{T} 4$ & $3(30)$ \\
\hline \multicolumn{2}{|l|}{ N stage } \\
\hline $\mathrm{N} 1$ & $1(10)$ \\
\hline $\mathrm{N} 2$ & $6(60)$ \\
\hline N3 & $3(30)$ \\
\hline \multicolumn{2}{|l|}{ Stage } \\
\hline III A & $6(60)$ \\
\hline III B & $4(40)$ \\
\hline \multicolumn{2}{|l|}{ Pathology } \\
\hline Adenocarcinoma & $7(70)$ \\
\hline Squamous cell carcinoma & $2(20)$ \\
\hline Undifferentiated carcinoma & $1(10)$ \\
\hline
\end{tabular}

Illustrated in table 2 is the comparison between the means of PTV before treatment (PTV1) and after adaptation (PTV2) for the ten patients involved in this study. The PTV2 was significantly smaller than PTV1 with an average reduction in the volume of $53 \%$.

The comparison between the conventional and adaptive planning as regards doses delivered to OARs is shown in table 2 .

There was statistically significant reduction in the maximum dose to the spinal cord, esophagus V35, esophagus V50, mean dose to the lung and lung V20. The average percentage reduction of lung parameters was higher than that of spinal cord and esophagus.

There was a reduction in the heart parameters (Dmax and Dmean); however, this reduction was not statistically significant.

\section{DISCUSSION}

Many trials reported shrinkage in the volume of locally advanced NSCLC during the course of radiation or concomitant chemoradiation ${ }^{7-10}$. In our study, CT imaging was repeated after $44 \mathrm{~Gy}$ and we observed an average reduction in PTV by about $50 \%$ which is in agreement with data reported by Britton et al and JuhlerNottrup et al ${ }^{11,12}$. Similar results of tumor repression have been observed by Guckenberger et al who reported a continuous tumor regression by $1.2 \%$ per day ${ }^{7}$. In contrast, Siker et al reported no clinically significant tumor regression in their series; however they used hypofractionated irradiation in their study ${ }^{13}$.

In the current study, the PTV after the first phase of radiotherapy (44 Gy) was redelineated and accordingly the radiotherapy treatment planning was recalculated. In the second phase the irradiated volume was reduced because of the reduction in the fields' sizes and the reshaping of each field. With this adaptive 3D conformal radiation plan, better conformality with significant decrease in the doses to the OARs was achieved.

The average reduction in mean lung dose and esophagus V50 was $24 \%$ and $11 \%$, respectively. Also adaptive plan technique resulted in reduced maximum spinal cord dose by $13 \%$.

In agreement with our results, Agrawal et al reported that mean lung dose decreased by $10.6 \%$ and esophagus V50 by $31 \%{ }^{14}$. Similar results had been observed by Guckenberger et al who reported a $5 \%$ reduction in mean lung dose with single-plan adaptation at week 3 or 5 . When they applied a twice-plan adaptation at weeks 3 and 5, the reduction in the mean lung dose was $5.6 \%$ and $7.9 \%$, respectively. ${ }^{7}$. Whereas Kataria et al achieved a higher reduction rate of $24 \%$ in mean lung dose with a single mid-treatment adaptive plan ${ }^{15}$. A similar study by Ding et al reported a significant reduction in V20 of the lung from $31.6 \%$ to $29.4 \%$ ( $p<0.001$ ), mean lung dose from 17.2 Gy to 15.9 Gy $(p=0.04)$ and spinal cord Dmax from 43.3 Gy to 40.2 Gy $(\mathrm{p}<0.001)^{16}$.

Adaptive radiotherapy planning has the potential for dose escalation thus improving local control which may be reflected on survival. Kong et al reported $3 \%$ reduction in risk of death with escalating dose of $1 \mathrm{~Gy}{ }^{17}$. Gillham et al performed a planning study in 10 patients 
Table 2. Reduction in the planning treatment volume (PTV) for the 10 patients

\begin{tabular}{llllll}
\hline Volume & PTV 1 & PTV2 & \% of reduction & $\boldsymbol{t}$ & $\boldsymbol{p}$ value \\
\cline { 1 - 3 } Mean \pm SD & $542.95 \pm 21.89$ & $256.79 \pm 28.13$ & $52.81 \pm 3.72$ & 70.125 & $<0.001$ \\
\cline { 1 - 2 } & $500-570$ & $200-290$ & $47.27-60$ & & \\
\hline
\end{tabular}

Table 3. Change in doses to organs at risk with adaptive plan

\begin{tabular}{|c|c|c|c|c|c|c|}
\hline Dose parameters & & Conventional plan & Adaptive plan & $\%$ of reduction & $t$ & $p$ value \\
\hline \multirow[t]{2}{*}{ Max. Spinal cord } & Mean \pm SD & $36.40 \pm 2.21$ & $31.60 \pm 2.18$ & $13.21 \pm 1.90$ & \multirow[t]{2}{*}{22.167} & \multirow[t]{2}{*}{$<0.001$} \\
\hline & Range & $33-39.4$ & $29-34.5$ & $10.53-16.22$ & & \\
\hline \multirow[t]{2}{*}{ Esophagus V35 } & Mean \pm SD & $30.82 \pm 3.35$ & $27.57 \pm 2.49$ & $10.36 \pm 2.70$ & \multirow[t]{2}{*}{8.616} & \multirow[t]{2}{*}{$<0.001$} \\
\hline & Range & $23-36.5$ & $21-30.2$ & $6.67-17.26$ & & \\
\hline \multirow[t]{2}{*}{ Esophagus V50 } & Mean \pm SD & $21.58 \pm 4.90$ & $19.26 \pm 4.93$ & $11.40 \pm 3.64$ & \multirow[t]{2}{*}{17.955} & \multirow[t]{2}{*}{$<0.001$} \\
\hline & Range & $11.8-29$ & $9.56-27$ & $6.9-18.98$ & & \\
\hline \multirow[t]{2}{*}{ Lung mean } & Mean \pm SD & $11.10 \pm 2.69$ & $8.12 \pm 0.96$ & $24.32 \pm 12.34$ & \multirow[t]{2}{*}{5.068} & \multirow[t]{2}{*}{0.001} \\
\hline & Range & $6.86-15.02$ & $6.3-9.7$ & $5.06-35.71$ & & \\
\hline \multirow[t]{2}{*}{ Lung V20 } & Mean \pm SD & $22.79 \pm 5.65$ & $17.92 \pm 3.48$ & $19.74 \pm 9.11$ & \multirow[t]{2}{*}{5.608} & \multirow[t]{2}{*}{$<0.001$} \\
\hline & Range & $12.47-29.43$ & $12-23.23$ & $3.77-36$ & & \\
\hline \multirow[t]{2}{*}{ Heart Max } & Mean \pm SD & $37.21 \pm 23.86$ & $37.16 \pm 23.81$ & $0.08 \pm 0.12$ & \multirow[t]{2}{*}{2.187} & \multirow[t]{2}{*}{0.057} \\
\hline & Range & $9.5-66.58$ & $9.5-66.5$ & $0-0.36$ & & \\
\hline \multirow[t]{2}{*}{ Heart Mean } & Mean \pm SD & $11.23+7.55$ & $11.19 \pm 7.59$ & $1.01 \pm 1.42$ & \multirow[t]{2}{*}{2.051} & \multirow[t]{2}{*}{0.071} \\
\hline & Range & $1.09-20.22$ & $1.07-20.2$ & $0-4$ & & \\
\hline
\end{tabular}

with advanced NSCLC using conventionally fractionated radiotherapy, $\mathrm{CT}$ imaging by $\mathrm{PET} / \mathrm{CT}$ were scheduled to be done after $50 \mathrm{~Gy}$ or $60 \mathrm{~Gy}{ }^{18}$. This adaptive radiotherapy technique allowed them to escalate the treatment dose from 70 to 74 Gy (5.7\% increase) on average. Feng et al acquired FDG PET-CT images after irradiation with $40-50$ Gy in 14 patients with NSCLC, using of single plan adaptation during the treatment course after 46 Gy lead to decrease in the mean lung dose by $10 \%$ on average and allowed the delivery of doses $>80$ Gy in 5 patients ${ }^{19}$.

Many studies reported the superiority of PET/CT when compared to CT scan in proper detection of the tumor and changing tumor staging with sensitivity and specificity of $64 \%$ and $74 \%$ respectively 20,21 . The incorporation of PET changed the treatment volumes in $58 \%$ of patients with NSCLC during treatment planning due to either upstaging or downstaging ${ }^{22}$. Thus, it seems profitable to use PET/CT in adaptive radiotherapy techniques. Adaptation of radiotherapy plans to the residual metabolic volume resulted in an $8 \%$ increase in tumor control probability ${ }^{23}$.

\section{Conclusion}

In NSCLC patients responding to the initial $44 \mathrm{~Gy}$, the adaptive radiotherapy technique used in our study can shrink the irradiated volume and reduce the dose delivered to OARs. The dose reduction may reach up to more than $30 \%$. The dose reduction in any OAR depends on the location of OAR with respect to the PTV. Adaptive planning resulted in more conformality, which can allow for dose escalation. This simple applicable technique can be easily implemented in limited resources centers.

\section{Conflict of inerest}

None to declare

\section{REFERENCES}

1- Ferlay J, Soerjomataram I, Dikshit R, et al. Cancer incidence and mortality worldwide: sources, methods and major patterns in GLOBOCAN 2012. Int J Cancer. 2015; 136(5): E359-386.

2- Robinson LA, Ruckdeschel JC, Wagner HJR, Stevens CW; American College of Chest Physicians. Treatment of non-small cell lung cancer-stage IIIA: ACCP evidencebased clinical practice guidelines ( $2^{\text {nd }}$ edition). Chest. 2007; 132(3 Suppl): 243S-265S.

3- Yuan S, Sun X, Li M, et al. A randomized study of involved-field irradiation versus elective nodal irradiation in combination with concurrent chemotherapy for inoperable stage III nonsmall cell lung cancer. Am J Clin Oncol. 2007; 30(3): 239-244.

4- Underberg RW, Lagerwaard FJ, Cuiipers JP, Slotman BJ, van Sornsen de Koste JR, Senan S. Four-dimensional CT scans for treatment planning in stereotactic radiotherapy for stage I lung cancer. Int J Radiat Oncol Biol Phys. 2004; 60(4): 1283-1290.

5- Spolestra FO, van Sornsen de Koste JR, Cuijpers JP, Lagerwaard FJ, Slotman BJ, Sensan S. Analysis of reproducibility of respiration- triggered gated radiotherapy for lung tumors. Radiother Oncol. 2008; 87(1): 59-64.

6- Guckenberger M, Wulf J, Mueller G, et al. Dose-response relationship for image-guided stereotactic body radiotherapy of pulmonary tumors: Relevance of 4D dose calculation. Int J Radiat Oncol Biol Phys. 2009; 74(1): 47-54.

7- Guckenberger M, Wilbert J, Richter A, Baier K, Flentje M. Potential of adaptive radiotherapy to escalate the radiation dose in combined radiochemotherapy for locally 
advanced non-small cell lung cancer. Int J Radiat Oncol Biol Phys. 2011; 79(3): 901-908.

8- Kupelian PA, Rmsey C, Meeks SL, et al. Serial megavoltage CT imaging during external beam radiotherapy for non-small-cell lung cancer: observations on tumor regression during treatment. Int J Radiat Oncol Biol Phys. 2005; 63(4): 1024-1028.

9- Bosmans G, van Baardwijk A, Dekker A, et al. Intrapatient variability of tumor volume and tumor motion during conventionally fractionated radiotherapy for locally advanced non-small-cell lung cancer: a prospective clinical study. Int J Radiat Oncol Biol Phys. 2006; 66(3): 748-753.

10- Fox J, Ford E, Redmond K, Zhou J, Wong J, Song DY. Quantification of tumor volume changes during radiotherapy for non-small-cell lung cancer. Int Radiat Oncol Biol Phys. 2009; 74(2): 341-348.

11- Britton KR, Starkschall G, Tucker SL, et al. Assessment of gross tumor volume regression and motion changes during radiotherapy for non-small-cell lung cancer as measured by four-dimensional computed tomography. Int J Radiat Oncol Biol Phys. 2007; 68(4): 1036-1046.

12- Juhler-Nottrup T, Korreman SS, Pedersen AN, et al. Interfractional changes in tumor volume and position during entire radiotherapy courses for lung cancer with respiratory gating and image guidance. Acta Oncol 2008; 47(7): 1406-1413.

13- Siker ML, Tome WA, Mehta MP. Tumor volume changes on serial imaging with megavoltage CT for non-small-cell lung cancer during intensity-modurated radiotherapy: how reliable, consistent, and meaningful is the effect? Int $\mathbf{J}$ Radiat Oncol Biol Phys. 2006; 66(1): 135-141.

14- Agrawal S, Kumar S, Maurya AK. Potential for adaptive dose escalation in radiotherapy for patients with locally advanced non-small-cell lung cancer in a low mid income setting. Br J Radial. 2017; 90(1070): 20140234.

15- Kataria T, Gupta D, Bisht SS, et al. Adaptive radiotherapy in lung cancer: dosimetric benefits and clinical outcome. Br J Radiol. 2014; 87(1038): 20130643.
16- Ding XP, Zhang J, Li BS, et al. Feasibility of shrinking field radiation therapy through $18 \mathrm{~F}-\mathrm{FDG}$ PET/CT after 40 Gy for stage III non-small cell lung cancers. Asian Pac J Cancer Prev. 2012; 13(1): 319-323.

17- Kong FM, Ten Haken RK, Schipper MJ, et al. High dose radiation improved local tumor control and overall survival in patients with inoperable/unresectable nonsmall-cell lung cancer: long-term results of a radiation dose escalation study. Int J Radiat Oncol Biol Phys. 2005; 63(2): 324-333

18- Gillham C, Zips D, Ponish F, et al. Additional PET/CT in week 5-6 of radiotherapy for patients with stage III nonsmall-cell lung cancer as a means of dose escalation planning? Radiother Oncol. 2008; 88(3): 335-341.

19- Feng M, Kong FM, Gross M, Fernando S, Hayman JA, Ten Haken RK. Using fluorodeoxyglucose positron emission tomography to assess tumor volume during radiotherapy for non-small-cell lung cancer and its potential impact on adaptive dose escalation and normal tissue sparing. Int J Radiat Oncol Biol Phys. 2009; 73(4): 1228-1234.

20- Gambhir SS, Czernin J, Schwimmer J, Silverman DH, Coleman RE, Phelps ME. A tabulated summary of the FDG PET literature. J Nucl Med. 2001; 42(5 Suppl.): 1S93S.

21- Bradley J, Thorstad WL, Mutic S, et al. Impact of FDG PET on radiation therapy volume delineation in nonsmall-cell lung cancer . Int J Radiat Oncol Biol Phys. 2004; 59(1): 78-86.

22- Erdi YE, Rosenzweig K, Erdi AK, et al. Radiotherapy treatment planning for patients with non-small-cell lung cancer using positron emission tomography (PET). Radiother Oncol. 2002; 62(1): 51-60.

23- Bral S, De Ridder M, Duchateau $M$, et al. Daily megavoltage computed tomography in lung cancer radiotherapy: correlation between volumetric changes and local outcome. Int J Radiat Oncol Biol Phys. 2011; 80(5): $1338-1342$ 